Théologiques

Théologiques

\title{
La Commission de vérité et réconciliation du Canada sur les pensionnats autochtones
}

\section{Bilan et prospective}

\section{Jean-François Roussel}

Volume 23, numéro 2, 2015

Théologies de la réconciliation

URI : https://id.erudit.org/iderudit/1042742ar

DOI : https://doi.org/10.7202/1042742ar

Aller au sommaire du numéro

\section{Éditeur(s)}

Faculté de théologie et de sciences des religions, Université de Montréal

\section{ISSN}

1188-7109 (imprimé)

1492-1413 (numérique)

Découvrir la revue

Citer cet article

Roussel, J.-F. (2015). La Commission de vérité et réconciliation du Canada sur les pensionnats autochtones : bilan et prospective. Théologiques, 23(2), 31-58. https://doi.org/10.7202/1042742ar

\section{Résumé de l'article}

La Commission de vérité et réconciliation du Canada (CVR) a traité abondamment du rôle des Églises dans le système des pensionnats autochtones et de leurs responsabilités pour surmonter cet héritage. La théologie est interpellée elle aussi par le rapport final de la CVR et par les implications de la " réconciliation ". Après avoir résumé l'histoire des pensionnats et expliqué l'origine de la CVR, nous rendons compte de ses résultats. Nous exposons les demandes que la CVR adresse spécifiquement aux Églises et aux coalitions oecuméniques. Nous examinons certaines critiques et mises en garde exprimées avant et pendant la CVR concernant le projet de réconciliation, les risques liés au mandat de la CVR et les aspects communicationnels des audiences. Nous engageons ensuite une réflexion théologique sur le thème de la réconciliation. En conclusion, nous proposons quelques pistes de réflexion pour le travail de la théologie après la CVR. 


\title{
La Commission de vérité et réconciliation du Canada sur les pensionnats autochtones
}

Bilan et prospective

\author{
Jean-François Roussel* \\ Institut d'études religieuses \\ Université de Montréal (Canada)
}

L'objectif de ce texte est de faire un bilan de la Commission de vérité et réconciliation du Canada sur les pensionnats autochtones (à l'avenir: CVR). La CVR a tenu des audiences à travers le Canada entre 2010 et 2014, avant de déposer son rapport final en 2015. Elle avait le mandat général de "contribuer à la vérité, à la guérison et à la réconciliation » (Iacobucci 2006) concernant l'héritage des pensionnats. Nous nous proposons de voir si et dans quelle mesure ce mandat a été rempli. Nous tenterons de situer les aspects ecclésiaux du dossier dans le cadre d'analyses anticoloniales du projet de réconciliation, proposées par des penseurs autochtones et par des chercheurs intéressés par les processus de justice transitionnelle.

Nous discuterons certaines critiques et mises en gardes exprimées avant et pendant la CVR, souvent par des penseurs autochtones. Ces critiques sont importantes, car elles mettent en relief les problèmes que peut poser un projet de réconciliation en contexte postcolonial. Nous serons

\footnotetext{
Jean-François Roussel est professeur agrégé à l'Institut d'études religieuses de l'Université de Montréal, où il enseigne en théologie contextuelle, particulièrement en rapport avec les questions autochtones. Il a récemment publié: (2016) «Lacombe's Chart. Imperialism and Spiritual Exile in the Indian Residential Schools of Canada ", dans G. Boodoo, dir., Religion, Human Dignity and Liberation, Sao Leopoldo, Oikos Editora; (2017) «Die schwierigen Wege zur Wahrheit und zur Versohnung. Kirchen und Theologie in Kanada nach Ende des Werziehungsanstalten für Kinder indigener Völker », Concilum, 52/3, p. 346-353.
} 
ensuite à même d'examiner les demandes de la CVR et tout particulièrement celles adressées aux Églises et aux coalitions œcuméniques. Ces demandes ne vont pas sans conséquences pour la théologie. Pour le moment, force est de constater que la théologie académique canadienne d'expression française n'a presque pas réfléchi sur la CVR et que la réflexion est à peine plus avancée au Canada anglais. Comment la théologie pourrait-elle répondre au projet de réconciliation souhaité par la CVR?

\section{L'histoire des pensionnats et de la CVR}

Il faut d'abord présenter l'origine de la CVR et son fonctionnement. On ne peut le faire sans résumer d'abord l'histoire des pensionnats, dont la complexité dépasse de loin le peu qu'il nous est possible de rapporter ici. À la fin du XIX ${ }^{e}$ siècle, le gouvernement du Canada et certaines dénominations chrétiennes formèrent un partenariat sous la forme d'un réseau national de pensionnats destinés à scolariser et à christianiser les enfants autochtones - à ces pensionnats s'ajoutent plusieurs écoles de jour dans des communautés autochtones. Les dénominations en question sont essentiellement des entités catholiques (communautés religieuses et quelques diocèses), l'Église anglicane, l'Église méthodiste et l'Église presbytérienne. À compter de 1925, l’Église méthodiste et la majeure partie de l'Église presbytérienne fusionnant pour créer l'Église Unie du Canada, celle-ci prit le contrôle des pensionnats méthodistes et de la majorité des pensionnats presbytériens. Il est largement reconnu que les pensionnats créèrent de profondes séquelles: déracinement culturel et linguistique, atteinte grave aux liens familiaux, violences psychologiques, physiques, sexuelles et spirituelles. Les archives conservées rapportent plus de 3000 décès, dont la cause n'est pas indiquée dans presque $50 \%$ des cas; s'y ajoutent probablement beaucoup plus de décès, dont la trace a disparu avec les dossiers des élèves et qu'on détruisait, à partir de 1935, cinq ans après le départ ou le décès de l'élève. Ces séquelles s'accumulèrent souvent sur plus d'une génération et elles se transmettent encore aujourd'hui, de génération en génération, dans les communautés touchées. Le tout dernier pensionnat ferma ses portes en 1998. Près de 150000 Autochtones auraient fréquenté ces établissements durant plus d'un siècle et des dizaines de milliers d'anciens pensionnaires sont encore en vie.

En 2006, à la suite de recours juridiques de survivants qui remontent aux années 1990, le gouvernement canadien, les représentants des survivants et les Églises ayant participé au système des pensionnats conclurent une «Convention de règlement relative aux pensionnats indiens». Selon 
les termes de cet accord juridique, les parties gouvernementales et ecclésiales prenaient divers engagements, dont celui de créer une Commission de vérité et réconciliation. Les objectifs, le mandat et les pouvoirs de la CVR ont été définis à l'annexe $\mathrm{N}$ de cette convention de règlement. Le mandat se formulait ainsi:

a) Reconnaître les expériences, les séquelles et les conséquences liées aux pensionnats; b) Créer un milieu holistique, adapté à la culture et sûr pour les anciens élèves, et leurs familles et collectivités, quand ils se présentent devant la Commission; c) Assister aux événements de vérité et de réconciliation, au niveau national et communautaire, et appuyer, promouvoir et faciliter de tels événements; d) Sensibiliser et éduquer le public canadien sur le système des pensionnats et ses répercussions; e) Repérer les sources et créer un dossier historique le plus complet possible sur le système des pensionnats et ses séquelles. Ce dossier doit être conservé et mis à la disposition du public, pour étude et utilisation future; f) Préparer et soumettre aux parties à la Convention un rapport, assorti de recommandations destinées au gouvernement du Canada, portant sur le système et l'expérience des pensionnats et présentant les aspects suivants: historique, objet, fonctionnement et supervision du système des pensionnats, effet et conséquences des pensionnats (notamment les séquelles systémiques, les conséquences intergénérationnelles et les effets sur la dignité humaine) et les séquelles permanentes de ces pensionnats; g) Appuyer la commémoration des anciens élèves des pensionnats et de leurs familles, conformément à la Directive sur la politique de commémoration. (Iacobucci 2006)

Les travaux de la CVR ont débuté en 2009 et les audiences publiques en 2010. De 2010 à 2014, sept «Évènements nationaux » ont eu lieu dans des grandes villes du Canada ainsi que des audiences régionales et un événement de clôture en 2015. Quiconque a eu l'occasion de participer à une ou à plusieurs de ses activités a pu mesurer l'intensité peu commune de ce qui s'y déroulait, dans une ambiance d'une grande dignité, et celle des échanges: témoignages bouleversants, écoute silencieuse et respectueuse de la part des représentants ecclésiaux, larmes et colère... expressions de dignité et d'amour des siens. Ce sont des moments inoubliables. Environ 6000 personnes ont été entendues, surtout des anciens pensionnaires ou des membres de leurs familles. Au nombre des activités incluses, les audiences comportaient des témoignages publics, des dépositions privées, des cercles de parole où des Autochtones prenaient la parole avant que des représentants institutionnels réagissent à leur tour de même que la possibilité pour des représentants des Églises, des institutions gouvernementales ou d'autres groupes de présenter des «expressions de réconciliation» 
publiques. Cela ne suffit pas à rapporter la richesse des échanges et des rencontres survenues dans ces rencontres faites de retrouvailles, d'entretiens privés et de libération personnelle.

Le rapport final compte plus de 4000 pages et a été publié en 2015. Dans un premier temps, on publie en juin le "sommaire ", qui compte à lui seul 594 pages; il s'agit d'une synthèse historique qui s'attarde aux séquelles des pensionnats et propose des "principes de la réconciliation ». Un troisième volume rapporte des témoignages de survivants (Commission de vérité et réconciliation du Canada 2015a; 2015b; 2015c). Les autres volumes, publiés six mois plus tard, sont beaucoup plus détaillés. Quatre d'entre eux portent sur l'histoire des pensionnats; un autre porte sur les enfants disparus et décédés au pensionnat; un autre expose les séquelles de l'expérience; le sixième volume porte sur la réconciliation; le dernier énonce les demandes de la CVR (Commission de vérité et réconciliation du Canada, 2015d; 2015e; 2015f; 2015g; 2015h; 2015i; 2015j; 2015k).

À long terme, quel sera l'héritage de la CVR ? A-t-elle atteint son double objectif de vérité et de réconciliation? Cette question est d'autant plus importante que des critiques ont été formulées au cours des dernières années, souvent par des voix autochtones. Qu'en est-il ?

\section{Le projet de réconciliation, une politique de distraction?}

Certaines critiques concernent les limites du mandat reçu par la CVR: elle ne peut "tenir des audiences formelles, faire fonction de commission d'enquête publique, $[. .$.$] mener un processus judiciaire formel »; elle n'a$ pas le "pouvoir d'assignation à témoigner ni le pouvoir de contraindre la présence ou la participation à l'un de leurs événements ou activités. La participation à tous les événements et activités de la Commission est à titre strictement volontaire $^{1}$ ». La CVR n'a pas le droit de dévoiler le nom d'une personne ayant commis un délit dans un pensionnat, sauf si celle-ci l'a déjà avoué ou si elle a déjà été condamnée pour ce délit ${ }^{2}$.

En 2010, le politologue Matt James, de l'Université de Victoria, spécialiste de la justice transitionnelle, soupèse les chances de réussite de la CVR. Il rappelle que de telles limites avaient été imposées à la commission

1. Commission de vérité et réconciliation du Canada, Notre mandat, art. 2, b.c.: $<$ http://www.trc.ca/websites/trcinstitution/index.php? p=19>.

2. Au début de chaque séance d'audiences publiques, les témoins étaient prévenus de ne pas nommer qui que ce soit si c'était pour l'accuser d'un acte criminel tel qu'un abus sexuel ou physique. 
semblable instituée au Chili (1990-1991), ce qui avait produit des résultats mitigés, car les membres de la junte militaire conservaient leur influence politique et avaient tout intérêt à ne pas être nommés (James 2010, 27). Jeff Corntassel (lui-même autochtone) et Cindy Holder font remarquer que la CVR est contrôlée par l'État lui-même, qui perpétue ainsi son rapport de force inéquitable avec les peuples autochtones (Corntassel et Holder 2008, 468). Ce que l'État souhaiterait en définitive, ce n'est pas la réparation mais la résolution du contentieux à son avantage propre, et qu'on «tourne la page », pour employer une expression rencontrée au début du

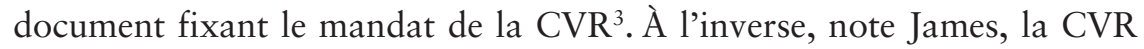
d'Afrique du Sud (1996-2003) jaugeait les actions et les responsabilités d'un régime qui appartenait au passé et qui ne contrôlait pas le processus (James 2010, 32).

À ce sujet, il faut préciser que ce n'est pas le gouvernement qui a institué la CVR mais un accord tripartite devant un juge. Il reste que le gouvernement n'est pas lié par les conclusions et les recommandations. Cela est évident quand on jauge l'attitude générale du gouvernement durant les travaux de la CVR. En 2008, exécutant une clause de la convention de règlement de 2006, le Premier Ministre a présenté des excuses formelles aux Premières Nations pour les abus survenus dans les pensionnats (Harper 2008). Par la suite, le gouvernement a fait preuve d'une attitude particulièrement arrogante dans divers dossiers touchant les Premières Nations, de même que d'une invariable indifférence aux travaux de la CVR. Cette attitude gouvernementale, les commissaires eux-mêmes la relèvent et en tirent une conclusion brutale: «[L]a promesse de réconciliation qui semblait sur le point de se réaliser en 2008 lorsque le premier ministre a présenté ses excuses aux survivants au nom de tous les Canadiens s'est cependant évanouie. » (Commission de vérité et réconciliation du Canada 2015b, 4) Si la franchise de cette protestation montre l'indépendance d'esprit des commissaires, elle confirme aussi la justesse des critiques - auparavant mentionnées.

Selon James, le mandat limité de la CVR pourrait empêcher celle-ci de faire toute la vérité sur les pensionnats. Cette appréhension est fondée dans la mesure où le rapport final s'abstient de désigner des nouveaux coupables ayant à répondre de leurs crimes allégués. Par contre, il jette un éclairage approfondi, sans précédent et sans complaisance, sur l'univers des pension-

3. Voir James (2010, 32); Commission de vérité et réconciliation du Canada, Notre mandat. 
nats. La responsabilité qu'il met en relief n'est pas individuelle mais nationale. Comment le Canada et des Églises ont-ils pu en arriver là ? Et comment pourront-ils s'amender aujourd'hui ? L'affirmation la plus remarquée du rapport est que le système des pensionnats a causé «un génocide culturel » (Commission de vérité et réconciliation du Canada 2015a, 1). Jugée réductrice par plusieurs Autochtones, elle a été catégoriquement rejetée par le gouvernement conservateur alors au pouvoir, qui craignait sans doute ses éventuelles utilisations par les Autochtones devant les tribunaux. Le rapport documente le colonialisme canadien et ajoute une pièce majeure au dossier des revendications autochtones.

Selon une autre critique formulée à propos de la CVR, le projet de réconciliation est une illusion. En 2009, avant le début des audiences publiques de la CVR, le philosophe mohawk Gerald Taiaiake Alfred formule son opposition au projet de réconciliation. Il rêve de «briser l'emprise» du projet de réconciliation sur les consciences des Autochtones et de le remplacer par un projet de justice:

It is my contention that reconciliation must be intellectually and politically deconstructed as the orienting goal of Indigenous peoples' political and social struggles. I see reconciliation as an emasculating concept, weakkneed and easily accepting of half-hearted measures of a notion of justice that does nothing to help Indigenous peoples regain their dignity and strength. One of my concerns in any discussion of reconciliation is finding ways to break its hold upon our consciousness so that we can move towards a true and lasting foundation for justice that will result in meaningful changes in the lives of Indigenous peoples and in the return of their lands. (Taiaiake 2009, 181)

Lors d'une conférence à Montréal en 2016, Alfred affirmait qu'aussi longtemps que le colonialisme persistera structurellement, le fait de se réconcilier avec la société canadienne reviendrait, pour les peuples autochtones, à se réconcilier avec le colonialisme. Pour Corntassel, le projet de réconciliation relève d'une "politique de distraction» (Corntassel 2012, 91.93-94). Les promoteurs de la réconciliation traitent le conflit comme le problème majeur à résoudre alors qu'il n'est en réalité qu'un effet de l'injustice permanente dont les peuples autochtones font les frais, soit celui d'être aliénés dans le pays dont ils sont dépossédés ${ }^{4}$. Denise Nadeau men-

4. On évoque les intérêts corporatifs qui invoquent la réconciliation pour tenter d'émousser la résistance de groupes autochtones à leurs projets d'exploitation territoriale (Corntassel, Chaw-Win-Is et T'lakwadzi 2009, 145). 
tionne aussi «un risque d'alibi» pour la société canadienne, qui pourrait être obnubilée par la découverte des abus dans les pensionnats au point de ne pas voir que ceux-ci découlent d'une réalité plus choquante encore:

Que nous le reconnaissions ou non, la relation avec les Premières Nations est injuste et déséquilibrée, du simple fait que nous résidons sur leur terre et que nous en tirons profit, alors que ce territoire leur a été volé, qu'il a été occupé et obtenu par des traités conclus dans une dynamique de pouvoir inégale et, qui plus est, souvent, ce territoire n'a pas été cédé par les peuples autochtones. (Nadeau 2012, 424)

Leanne Simpson, théoricienne et écrivaine anishinabé du sud de l'Ontario, formule une critique semblable:

But the idea of reconciliation is not new. Indigenous Peoples attempted to reconcile our differences in countless treaty negotiations, which categorically have not produced the kinds of relationships Indigenous peoples intended. I wonder how we can reconcile when the majority of Canadians do not understand the historic of contemporary injustice of dispossession and occupation, particularly when the state has expressed its unwillingness to make any adjustment to the unjust relationship. (Simpson 2011, 21)

L'appel à la réconciliation, maintes fois entendu à travers le pays, Simpson le compare à « une relation abusive où une personne est abusée physiquement, émotionnellement, spirituellement et mentalement. Elle veut sortir de la relation, mais au lieu de la supporter, nous sommes tous autour de l'abuseur, parce qu'il veut se “réconcilier”. » (Simpson 2011, 21) L'abuseur, pour sa part, ne remet pas en question son attitude abusive: ce qu'il désire, c'est résoudre son malaise: s'excuser pour se sentir moins coupable.

Ces critiques sont essentielles, car la tentation est grande de réduire la réconciliation à un moyen pour dissiper l'inconfort des colonisateurs. Cependant, le rapport de la CVR dépasse de loin une compréhension de la réconciliation comme pardon pour les injustices passées et présentes. La réconciliation est indissociable d'un processus de décolonisation: «Le système des pensionnats et son héritage doivent être placés dans le contexte international élargi des politiques coloniales qui précèdent l'établissement des écoles et se poursuivront après leur fermeture. » (Commission de vérité et réconciliation du Canada 2015e, 640) La CVR définit la réconciliation comme « un processus continu [nous soulignons] visant à établir et à main-

5. Voir Commission de vérité et réconciliation du Canada (2015d, 14). 
tenir des relations respectueuses. Un élément essentiel de ce processus consiste à réparer le lien de confiance en présentant des excuses, en accordant des réparations individuelles et collectives, et en concrétisant des actions qui témoignent de véritables changements sociétaux. » (Commission de vérité et réconciliation du Canada 2015e, 640) La CVR rappelle que ces «changements sociétaux " étaient déjà recommandés par la Commission royale sur les peuples autochtones: "reconstitution» des nations autochtones ainsi que de leurs pouvoirs, nouvelle répartition des territoires et de leurs ressources, formation des Autochtones en vue de l'autodétermination, leviers économiques réels. Ils font partie intégrante de la réconciliation, affirme la CVR (Commission de vérité et réconciliation du Canada, 2015j, 25-26). Évoquant la rupture du lien de confiance entre les peuples autochtones et la société canadienne, de même que la nécessité de restaurer cette confiance en reconnaissant "pleinement le droit à l'autodétermination des peuples autochtones dans le cadre d'un partenariat avec une souveraineté canadienne viable », le rapport poursuit:

La réconciliation doit devenir un mode de vie. Il faudra de nombreuses années pour réparer les relations et les liens de confiance rompus dans les communautés autochtones et entre les Autochtones et non-Autochtones. La réconciliation nécessite non seulement des excuses, des réparations, un réapprentissage de l'histoire nationale du Canada et une cérémonie commémorative publique, mais également de véritables changements sociaux, politiques et économiques. La sensibilisation du public et le dialogue permanents sont essentiels à la réconciliation. Les gouvernements, les Églises, les institutions d'enseignement et les Canadiens de tous les milieux ont la responsabilité d'agir de façon concrète pour la réconciliation, en collaboration avec les peuples autochtones. La réconciliation est la responsabilité de chacun d'entre nous. (Commission de vérité et réconciliation du Canada 2015b, 194-195)

\section{L'effet public de la CVR, entre objectif et résultat}

D’autres critiques s'intéressent aux aspects communicationnels, pragmatiques et subjectifs de la CVR ainsi qu'à leurs effets à long terme. Elles décèlent un décalage entre l'effet recherché sur la population et l'effet réel. Il est intéressant de remarquer l'un des éléments figurant au mandat de la CVR: "Sensibiliser et éduquer le public canadien sur le système des pensionnats et ses répercussions "; cet objectif s'est avéré superflu dans la plupart des commissions similaires ailleurs dans le monde, car les faits 
examinés y ont touché une large part de la population. De la part de la société dominante, James prévoit l'absence d'intérêt à examiner lucidement l'histoire d'un système qui révèle crûment le colonialisme canadien. Les Autochtones possèdent-ils le rapport de force qui leur permettrait de contraindre cette société à faire cet examen? Certainement pas, selon l'auteur, ni en termes démographiques ni en termes de crédibilité. En comparaison, la commission comparable instituée en Argentine après la fin de la dictature jouissait d'un très fort appui populaire, ce qui s'explique largement par le fait que la majorité de la population s'identifiait aux victimes de la dictature (James 2010, 26).

La CVR a voulu appliquer des règles de la justice réparatrice vécue traditionnellement dans les peuples autochtones; cela implique la prise de parole des victimes et les accusés doivent aussi s'exprimer pour reconnaître leurs torts, en cherchant comment les réparer. Cependant, Ronald Niezen, un anthropologue du droit de l'Université McGill, étudie le déroulement de la CVR canadienne. Il soumet une hypothèse troublante: la logique discursive et le fonctionnement de la CVR auraient produit des "solitudes » : des «survivants ", pour reprendre l'appellation consacrée et réifiante, puis des abuseurs gouvernementaux et ecclésiaux; ces deux camps sont appelés à s'exprimer selon deux registres discursifs assez rigides et ne souffrant guère de dérogation. Ces deux solitudes, Niezen les définit comme «ces formes de savoir et d'appartenance, dans certains cadres légaux particuliers, qui s'expriment les unes après les autres en créant des conditions pour des comportements conformes et des croyances uniformes. » (Niezen 2013, 151)

Le tout se déroulerait sous l'œil distant d'une population canadienne étrangère au drame rapporté par médias interposés (Niezen 2013, 151s). Assez paradoxalement, dirions-nous, cette expression d'une "peine indescriptible, tissée à l'aune du quotidien d'un si grand nombre de peuples autochtones, que la plupart des peuples non autochtones ne veulent ni voir ni même imaginer ", comme l'écrit Nadeau (2012, 426), serait formatée, selon Niezen, et à terme neutralisée: le public non autochtone finirait par ne plus l'entendre que comme répétition du même, comme «banalité du mal» (Niezen 2013, 150). Nous sommes bien loin alors de la justice réparatrice. Peu de place étant laissée à des expressions dérogeant à ce schéma binaire, Niezen voit mal comment celui-ci peut favoriser un rapprochement, prélude à une réconciliation. Il relève l'amertume de gens d'Église essuyant un opprobre généralisé, tancés par l'audience quand ils se risquent à présenter certains aspects positifs de leur expérience des pensionnats. 
C'est ce que l'oblat Tom Cavanaugh a voulu présenter comme «ma vérité », avant d'être chahuté sans ménagement par l'assistance alors qu'il faisait l'éloge du pensionnat où il avait œuvré (Niezen 2013, 89-94).

La suite des choses a-t-elle donné raison à James et Niezen? Le régime de production de la vérité à la CVR a-t-il renforcé les deux solitudes? Le public canadien semble avoir été peu réceptif aux travaux de la CVR. Cela a été entendu régulièrement dans les activités publiques de la commission, qui occupaient peu d'espace dans les médias ${ }^{6}$. Les audiences publiques n'ont pas été diffusées à la télévision; on a rarement entendu des témoins s'exprimer dans les reportages télévisés et peu d'émissions d'affaires publiques se sont intéressées à l'avancement des travaux ou à en présenter des analyses.

La convergence des témoignages renforce leur crédibilité mais, selon l'analyse de Niezen, leur similarité finirait par désensibiliser une part du public au lieu de maintenir son empathie. Il est bien difficile d'établir si cette critique est fondée, si cet effet l'a emporté sur l'effet de sensibilisation et d'empathie recherché auprès de la population canadienne.

Cependant, il faut noter que le registre discursif choisi par la CVR a été emprunté à la pratique de la justice réparatrice valorisée et traditionnellement pratiquée chez les Autochtones: aucune réconciliation n'est

6. Une recherche par mots-clés sur la base de données Eureka, dans toute la presse écrite francophone du Canada, montre qu'à partir du début des audiences de la Commission en juin 2010, les articles comportant en introduction les termes "Commission de vérité et réconciliation" et "autochtones» étaient déclassés de loin par d'autres thèmes tels que la Commission d'enquête sur la corruption dans l'industrie de la construction dans les mêmes sources (un événement non pas national, pourtant, mais limité au Québec) et les "accommodements raisonnables". La CVR n'arrive à se démarquer qu'à partir de 2015-2016, peut-être parce que le nouveau gouvernement libéral fait plusieurs annonces relatives au suivi des demandes de la Commission.

\begin{tabular}{|c|c|c|c|}
\hline & $\begin{array}{c}\text { Commission de vérité } \\
\text { et réconciliation } \\
\text { + autochtones }\end{array}$ & $\begin{array}{c}\text { Commission d'enquête } \\
\text { sur la corruption dans } \\
\text { l'industrie de la } \\
\text { construction }\end{array}$ & $\begin{array}{c}\text { Accommodements } \\
\text { raisonnables }\end{array}$ \\
\hline $2010-2011$ & 93 & 3 & 601 \\
\hline $2011-2012$ & 110 & 381 & 488 \\
\hline $2012-2013$ & 260 & 1264 & 715 \\
\hline $2013-2014$ & 134 & 503 & 1564 \\
\hline $2014-2015$ & 153 & 433 & 434 \\
\hline $2015-2016$ & 579 & 495 & 334 \\
\hline
\end{tabular}


possible si l'offenseur ne reconnaît pas sincèrement les torts causés ainsi que leurs séquelles sur les victimes; cette reconnaissance n'est pas le lieu pour présenter des réfutations ou des circonstances atténuantes. Les audiences ont aussi été l'occasion de souligner les gestes positifs de plusieurs membres du personnel des pensionnats. Le rapport en fait régulièrement état, malgré sa condamnation générale d'un système "sévère et souvent destructeur ${ }^{7}$ ». Par exemple, le témoignage du père Cavanaugh (cité quelques fois dans le rapport) est rapporté ainsi que la réplique accusatrice d'une ancienne pensionnaire de l'établissement concerné, juste après celui de l'Oblat. À cet égard, le rapport parle de «deux vérités apparemment inconciliables " (Commission de vérité et réconciliation du Canada 2015e, 594-595.598). Comment «honorer la vérité »? Le rapport final reconnaît qu'il a été impossible pour les Églises et les membres du personnel de faire entendre leurs éventuelles divergences, ce qu'il explique par les émotions encore vives qui parcouraient les assistances (Commission de vérité et réconciliation du Canada 2015a, 18). Reprenons les propos d'une religieuse citée dans le rapport, qui avait travaillé dans un pensionnat:

Je crois qu'il serait utile pour les personnes qui ont connu les pensionnats indiens d'entendre personnellement les histoires. Et je crois aussi que ce serait utile, lorsque cela est approprié... [pour] les anciens élèves qui sont sur la voie de la guérison... d'entendre quelques-unes de nos histoires, ou d'entendre quelques-uns de nos points de vue. Mais je sais que c'est une chose très difficile à faire... Sûrement, ceci n'est pas le bon moment pour essayer de demander à tous les anciens élèves de s'asseoir et d'écouter la logique des anciens membres du personnel, parce qu'il y a trop d'émotions dans cela... et il y a trop peu de confiance... on ne peut faire ce genre de choses quand les niveaux de confiance sont faibles. Alors, je pense que vraiment, une chose très importante pour les anciens membres du personnel est d'écouter les histoires et d'être assez courageux pour se contenter de les écouter... Là où des torts ont été faits, où des abus sont survenus, où les punitions étaient extrêmes, et partout où des agressions sexuelles sont survenues, nous devons quelque part avoir le courage de nous asseoir et d'en parler, et de nous excuser. Je ne sais pas comment cela arrivera. (Commission de vérité et réconciliation du Canada 2015b, 122)

7. Ainsi, «dès le début, les Affaires indiennes et les Églises placent leurs propres intérêts devant ceux des enfants dont ils ont la garde et camouflent lâchement les actes dont les enfants sont victimes. " (Commission de vérité et réconciliation du Canada 2015e, 607). Pour des observations positives sur le travail d'employés, voir Commission de Vérité et réconciliation du Canada (2015a, 109). 
Relevons deux groupes de critiques opposées: celles qui contestent le projet de réconciliation au nom d'une dure vérité selon laquelle la réconciliation sans une profonde décolonisation est une illusion et celles qui redoutent une entrave à la réconciliation par une certaine standardisation des témoins (survivants vs abuseurs) et des paroles acceptables. Les deux critiques s'articulent bien l'une à l'autre dans la mesure où, dans les deux cas, on craint que la CVR ne soit vécue comme un point terminal, comme l'avortement du processus de transformation sociale et subjective. Les réponses qui seront données aux demandes finales de la CVR permettront de voir le caractère durable de l'engagement pour la réconciliation, et d'une réconciliation au sens où l'entend la Commission.

\section{Les demandes de la CVR aux Églises et aux coalitions œcuméniques}

La CVR formule 94 recommandations, ou plutôt "demandes ", à l'ensemble des institutions canadiennes: gouvernements, écoles, Églises et médias (Commission de vérité et réconciliation du Canada 2015k). La diversité des champs concernés montre bien l'ampleur des enjeux touchés par l'héritage des pensionnats: promulgation d'un «pacte de réconciliation" entre le gouvernement canadien et les peuples autochtones; demandes en matière d'éducation, de revitalisation des langues autochtones, de santé, de justice, de traitement des revendications territoriales autochtones, de sensibilisation de la population canadienne à l'histoire des Premières Nations et à celle des pensionnats, à l'école comme dans les médias, auprès des personnes immigrantes. On demande aussi un plan d'action global pour atteindre tous ces objectifs ainsi qu'un bilan périodique de sa mise en œuvre. À cet effet, la Commission demande la création d'un Conseil national de réconciliation. Et avant tout, elle demande à l'ensemble des institutions du Canada, gouvernementales, civiles et ecclésiales, l'adoption d'un principe axiologique: "mettre en œuvre la Déclaration des Nations Unies sur les droits des peuples autochtones dans le cadre de la réconciliation" (à l'avenir: DNUDPA) (Commission de vérité et réconciliation du Canada 2015k). L'étendue des demandes correspond à celle du colonialisme dont les pensionnats ne sont qu'une facette. Examinons les demandes que la CVR adresse spécifiquement aux Églises et aux coalitions œcuméniques.

Il est demandé aux Églises et aux coalitions œcuméniques d'adopter et de promouvoir les principes et les normes de la DNUDPA (demande 48). Les quatre Églises parties à la convention de 2006 ont répondu favorable- 
ment à cette demande. Cependant, la déclaration en question, adoptée en 2007 par l'Assemblée générale des Nations Unies, concerne d'abord les États. En quoi les Églises et les coalitions œcuméniques seraient-elles visées? Il s'agit peut-être de la demande dont les conséquences seraient les plus fondamentales.

La DNUDPA affirme les droits «spirituels» des peuples autochtones. Ils incluent la restitution de territoires et d'objets de patrimoine religieux, le «droit de manifester, de pratiquer, de promouvoir et d'enseigner leurs traditions, coutumes et rites religieux et spirituels; le droit d'entretenir et de protéger leurs sites religieux et culturels et d'y avoir accès en privé; le droit d'utiliser leurs objets rituels et d'en disposer " (Commission de vérité et réconciliation du Canada 2015k; Organisation des Nations Unies 2007). Ces droits spirituels ne sont-ils pas déjà reconnus? Les assauts contre les traditions spirituelles autochtones, s'ils sont encore pratiqués par certaines dénominations fondamentalistes jusqu'au cœur de communautés autochtones, ont largement cessé dans la plupart des Églises du Canada, tandis que les coalitions œcuméniques sont tolérantes par définition. Cependant, comme le rapport de la CVR le fait remarquer, la tolérance des Églises classiques ne suffit pas: elles doivent dénoncer les fondamentalistes, qui s'attaquent à des pratiques dont l'efficacité pour la guérison des survivants est démontrée. Elles doivent le faire pour soutenir les Autochtones traditionalistes dans leur travail de guérison. Elles doivent aussi dire aux survivants restés chrétiens et craignant de recourir aux pratiques spirituelles autochtones de guérison qu'ils ont le droit de le faire. "Avoir un droit qu'on a peur d'exercer équivaut à ne pas avoir ce droit du tout. » (Commission de vérité et réconciliation du Canada 2015b, 118)

Toutes les confessions religieuses au Canada doivent respecter ce droit, et les Églises Unie, anglicane, presbytérienne et catholique, qui sont parties à la Convention de règlement, ont une responsabilité particulière quant à la reconnaissance officielle de la spiritualité autochtone comme forme valide de culte qui est égale à la leur. Il ne revient pas à des personnes individuelles au sein des Églises de lever la voix quand la liberté de culte est niée. En fait, ce sont aux Églises, en tant qu'institutions religieuses, d'affirmer la valeur de la spiritualité autochtone en tant que telle. Sans cette reconnaissance officielle, un rapprochement complet et durable demeurera impossible. La guérison et la réconciliation ont une dimension spirituelle dont les Églises doivent prendre conscience, en partenariat avec les chefs spirituels autochtones, les survivants, leurs familles et les communautés. (Commission de vérité et réconciliation du Canada 2015b, 118) 
Lors d'un colloque à Chicago en 2016, Eva Solomon, Ojibway de l'île Manitoulin, religieuse catholique et figure bien connue de l'inculturation autochtone du christianisme, a affirmé que s'il est vrai que l'Église catholique dit oui à la DNUDPA, elle ne comprend pas réellement à quoi elle dit oui. Cette remarque mérite réflexion.

Au nombre des implications mentionnées par le libellé de la demande 48.ii de la CVR, les Églises sont appelées à «respecter le droit à l'autodétermination des peuples autochtones dans les cas d'ordre spirituel» (ONU 2007, 12.1). Or, la DNUDPA affirme à quelques reprises le droit des peuples autochtones au consentement libre et éclairé pour les projets qui les touchent, particulièrement en matière territoriale mais ultimement en toute matière qui les concerne; ce droit s'exerce dans une négociation avec les instances propres des Autochtones (ONU 2007, 19). Qu'en est-il de ce droit sur le plan spirituel et notamment dans les Églises? Plus spécifiquement dans l'Église catholique, peut-on dire que les Autochtones disposent de leviers pour exercer leur droit à l'autodétermination? On n'y trouve aucune instance autochtone autre que consultative. Lors du colloque précédemment mentionné, la théoricienne postcoloniale mi'kmaq Marie Baptiste déclarait avec force que les peuples autochtones n'ont pas besoin de prêtres blancs venant chez eux parés d'ornements autochtones mais dont le leadership reproduit structurellement le colonialisme spirituel. Cette remarque n'a pas manqué de susciter un certain malaise parmi l'auditoire, mais Baptiste poursuivit: ce qu'il faut, dit-elle, ce sont des pratiques de décolonisation par des Autochtones, où ceux-ci pourraient élaborer, dans l'Église, leurs discours et leurs positions communes à partir de leurs patrimoines et de leurs visions du monde.

La réconciliation entre Autochtones et non-autochtones impliquerait, selon la CVR, d'écouter et de valoriser les savoirs des aînés autochtones ainsi que leurs systèmes de savoir: «Appuyer la revitalisation culturelle des peuples autochtones et intégrer les systèmes de savoir, les histoires orales, les lois, les protocoles et les liens avec la terre des Autochtones sont des éléments essentiels au processus de réconciliation. » (Commission de vérité et réconciliation du Canada 2015b, 130) Le droit à l'autodétermination en matière spirituelle implique des aménagements non seulement symboliques mais concrets. Les obstacles sont considérables, notamment en raison des règles canoniques de gouvernance de l'Église catholique. En contexte autochtone, ces règles formelles confortent la marginalisation des Autochtones; sera-t-il possible d'y remédier un jour?

Outre les droits spirituels, la DNUDPA énonce les droits des peuples autochtones dans de multiples domaines: territoriaux, politiques, culturels, 
linguistiques, éducatifs, etc. Les Églises peuvent la prendre comme charte de leurs appuis aux Autochtones dans une perspective de pastorale sociale.

La CVR demande aussi aux Églises et aux groupes interconfessionnels «qui ne l'ont pas déjà fait » de "répudier » les concepts utilisés pour justifier l'impérialisme, notamment la Doctrine de la Terra Nullius et la Doctrine de la Découverte (demande 49). Cette répudiation est aussi demandée au gouvernement canadien lors de la promulgation du futur pacte de réconciliation (demande 45). Il s'agit d'un ensemble de textes papaux du $x^{\mathrm{e}}$ siècle, au premier chef les bulles papales Dum diversas (1452), Romanus Pontifex (1455) et Inter Caetera (1493) affirmant le droit des puissances chrétiennes de "vaincre» et de "soumettre " les peuples non chrétiens afin de prendre possession de leurs terres, dites «terres de personne» (nullius). Comme l'affirme à ce sujet un document de l'Instance permanente sur les questions autochtones des Nations Unies, "[1]'usage de la violence et des conversions forcées pour "soumettre" les non-chrétiens a fait le lit de la domination et de l'asservissement des peuples autochtones. » (Conseil économique et social 2010a) Aux dires du Saint-Siège, ces doctrines ont été annulées peu de temps après leur fabrication (Conseil économique et social 2010b); c'est aussi ce que soutient un document de travail de la Commission pour la Justice et la Paix de la Conférence des évêques catholiques du Canada, qui offre une synthèse bien documentée (Commission pour la justice et la paix, 2016). Néanmoins, ces doctrines ont essaimé rapidement et de manière durable dans la vision du monde européenne, au-delà de l'Église catholique et des Églises. Désormais intégrées dans la jurisprudence des États, elles sont encore considérées par les tribunaux dans l'étude des revendications territoriales des peuples autochtones, au détriment de ces derniers. Selon la CVR, elles sont au fondement de l'impérialisme dont découlent les pensionnats autochtones. D’ailleurs, cet impérialisme a été confessé par des entités chrétiennes ayant présenté des excuses aux Autochtones pour les pensionnats ${ }^{8}$. Au-delà de la caducité de ces doctrines, qui apparaît la plupart du temps comme conséquence implicite d'une série de gestes à travers l'histoire, nous croyons que le Saint-Siège apporterait une contribution significative en faisant une déclaration officielle de répudiation de ces doctrines, comme l'a fait à son échelle la Conférence des évêques catholiques du Canada dans le document que nous venons de mentionner. Il faut souligner l'importance que la CVR accorde à la dimension visible de la réconciliation, sous la forme de la

8. Par exemple, les Oblats, dans leur déclaration de 1991 (Crosby 1991, 2.3-4). 
commémoration publique et du "partage de la vérité " (Commission de vérité et réconciliation du Canada 2015b, 129). Par exemple, dans les évènements de la CVR, les «expressions de réconciliation» formulées par les représentants de la société canadienne étaient déposées dans un magnifique coffre de bois, et il est prévu qu'elles y demeurent, en mémoire des paroles dites. C'est pourquoi une déclaration officielle d'abrogation de la Doctrine de la Découverte par le Saint-Siège constituerait une contribution à la réconciliation.

La CVR demande aussi au pape de présenter des excuses officielles, si possible lors d'une visite au Canada, avant le $1^{\text {er }}$ juin 2016, pour les mauvais traitements et abus survenus dans les pensionnats catholiques (demande 58). Jusqu'à ce jour, l'épiscopat catholique canadien résiste à l'idée d'assumer une responsabilité morale relativement à des institutions auxquelles peu de diocèses ont collaboré et qui étaient plutôt gérées par des congrégations religieuses. Ce raisonnement épiscopal vaut-il aussi pour le pape? Le 29 avril 2009, Benoît XVI avait exprimé auprès de représentants des Premières Nations «sa peine face à l'angoisse causée par la conduite déplorable de certains membres de l'Église» dans les pensionnats, offrant «sa sympathie et sa solidarité dans la prière» (Saint-Siège 2009, 389). Le rapport de la CVR questionne sèchement la pertinence cette déclaration, en reprochant au pape de refuser d'assumer son autorité spirituelle et morale sur l'ensemble du monde catholique (Commission de vérité et réconciliation du Canada 2015j, 111-112). Le $1^{\text {er }}$ juin 2016, les excuses demandées par la CVR n'avaient pas encore été présentées. De passage en Bolivie, le 9 juillet 2015, donc peu après la publication des demandes de la CVR, le pape François a demandé pardon aux peuples autochtones: «de nombreux et de graves péchés ont été commis contre les peuples originaires de l'Amérique au nom de Dieu. [...] je demande humblement pardon, non seulement pour les offenses de l'Église même, mais pour les crimes contre les peuples autochtones durant ce que l'on appelle la conquête de l'Amérique. » (Pape François 2015) Actuellement, des représentants du Vatican affirment que le pape envisage de venir au Canada pour présenter des excuses aux Autochtones dans un avenir proche. L'avenir dira ce qu'il en est.

La CVR demande aussi aux Églises d'élaborer des stratégies d'éducation sur «le rôle joué par l'Église» dans le colonialisme et dans les pensionnats (demande 59) ainsi qu'un programme de formation à propos de ces matières, à l'intention du personnel ecclésial (demande 60). Pour ces deux demandes, il est souhaitable que les centres de formation théologique se mobilisent. 
En outre, la CVR demande la création d'un «fonds permanent» pour soutenir des projets autochtones de guérison, de revitalisation linguistique, culturelle et spirituelle (demande 61). De quelle ampleur pourrait être ce fonds? Elle ne peut être que proportionnelle à l'état des finances ecclésiales. Il faut rappeler que la constitution d'un fonds pour supporter des projets de guérison, prévue par la Convention de règlement de 2006, avait permis d'amasser un montant de 100 millions de dollars, aujourd'hui épuisé. Il fut affecté à la Fondation autochtone de guérison, pour financer des projets de guérison dans les communautés autochtones. La demande 61 pose tout de même une question à l'Église. La réconciliation implique une réparation. À cet égard, on peut se réjouir que l'Église ait généralement mis fin à son attitude hostile envers les rites et les croyances autochtones. Pourtant, force est de constater l'effet dévastateur de cette longue répression sur ce patrimoine; les Autochtones s'efforcent aujourd'hui d'en récupérer la part occultée, souvent en collaboration avec des anthropologues et des historiens. Comment les Églises et les coalitions œcuméniques pourraient-elles contribuer à revaloriser ces traditions? Ce pourrait être par exemple dans l'organisation de rencontres interreligieuses, dans le soutien aux initiatives culturelles et spirituelles même quand elles ne sont pas liées au christianisme: une forme d'hospitalité qui, à vrai dire, ne serait que juste retour des choses, car l'Église canadienne n'aurait jamais pu naître sans l'hospitalité des Autochtones non chrétiens.

Enfin, les demandes 73 et 74 sont poignantes: il est demandé au gouvernement et aux Églises de collaborer pour créer un registre des cimetières et, dans la mesure du possible, des sépultures qui s'y trouvent, puis d'informer les familles et les communautés des lieux de sépulture des enfants en question. On sait que des milliers d'enfants sont décédés dans les pensionnats et que, dans bien des cas, les familles n'ont jamais été informées des circonstances des décès ni des lieux de sépulture.

\section{Ouvertures pour un projet théologique}

Mark MacDonald est ojibway, évêque national autochtone de l'Église anglicane du Canada et théologien. Selon lui, si en général les Églises ont présenté des excuses dans le système des pensionnats puis ont entrepris des transformations de leurs pratiques, il leur reste encore à développer une théologie et des perspectives spirituelles à partir de cette expérience historique (MacDonald 2016, 6). Nous ne nous trouvons qu'au tout début de ce chantier, qui est d'importance majeure. Peu de théologiennes et théolo- 
giens ont entrepris ce travail (Nadeau 2012; Baum 2014; Andraos 2015; Leblanc 2016; Roussel 2016; Roussel [s.d.]).

La théologie académique canadienne d'expression française a prêté peu d'attention aux questions autochtones en général. Sur la question autochtone, Achiel Peelman a joué un rôle majeur et peut être considéré comme un pionnier. En plus de plusieurs articles, il a consacré deux monographies à cette question (Peelman 1992; Peelman 2014). J'insiste sur deux de ses idées. D'abord, le temps est venu de la mission renversée, où on se mettrait à l'école des Autochtones. Ensuite, dans ce pays qui est avant tout le leur, les Autochtones doivent devenir des interlocuteurs des théologiennes et des théologiens. Il faut bien constater que les travaux de Peelman ont trouvé peu d'écho dans la communauté théologique canadienne francophone. Un peu comme si la question autochtone n'avait été au fond qu'un thème marginal, une subdivision thématique de la missiologie. Pourtant, combien de questions qui intéressent la théologie s'y entrecroisent: dialogue interculturel et inculturation, droits humains et libération, écothéologie, ritualité, pour ne nommer que celles-là !

\section{Engager une pratique dialogale avec des Autochtones}

Comment faire théologie non pas sur les Autochtones mais avec eux, en accord avec les principes de la DNUDPA ? Où sont les Autochtones en théologie, demandera-t-on? S'il existe une littérature autochtone en théologie, au Canada mais surtout aux États-Unis ${ }^{9}$, elle n'est pas très abondante. Au Canada français, elle est inexistante. Cela tient sans doute en partie au faible poids démographique des communautés autochtones, conjugué à la taille relativement restreinte de la communauté théologique si on la compare à celle d'autres disciplines. La situation s'explique aussi en partie par le niveau de scolarisation moyen chez les peuples autochtones, plus bas que parmi l'ensemble de la population; toutefois, on rencontre des Autochtones dans d'autres disciplines, l'anthropologie par exemple. Enfin, si la théologie semble perçue comme forme doctrinale et savante de la religion, cette dernière trouve aux yeux des Autochtones son incarnation première dans des Églises qui sont précisément au cœur de l'héritage violent des pensionnats. Enfin, la notion de religion, considérée comme espace sacré et distingué d'un espace profane, ne suscite guère d'intérêt chez beaucoup d'Autochtones, à l'exception des personnes plus âgées.

9. Par exemple: Deloria Jr (2003); Donaldson (2002); Kidwell, Noley et Tinker (2001); Mackay (2003, 175); Leblanc et Leblanc (2011); Treat (1996); Weaver (1998). 
Pourtant, nombreux sont les auteurs autochtones qui font, à leur manière, ce que la théologie chrétienne fait aussi: transmettre les traditions (d'abord orales) en les prenant non pas comme objets d'étude mais comme référence herméneutique, pour interpréter la réalité et produire avec créativité des chemins de vie possible ${ }^{10}$. C'est le cas de Taiaiake Alfred, qui articule une pensée politique en articulant les cérémonies iroquoises et l'analyse politique décoloniale, et de Leanne Simpson, qui se nourrit à la vision du monde racontée par les Aîné-e-s de son peuple (Alfred 2005; Simpson 2011). Ce ne sont que deux exemples d'une littérature autochtone déjà bien établie. On y trouve de nombreuses œuvres porteuses de souffle et de vision, même si elles ne se reconnaissent pas dans le concept de théologie, sauf exception. En outre, antérieurement aux auteurs et aux œuvres littéraires, les traditions en question sont transmises et s'actualisent par l'oralité et par des Aînés généralement éloignés de la culture académique. Il s'agit, pour les théologiennes et théologiens, de devenir des interlocuteurs de ces sagesses qui appréhendent le monde dans sa globalité.

\section{Déconstruire l'ordre socio-symbolique colonial au-delà des pensionnats}

Achiel Peelman rappelle l'injustice permanente qui frappe les peuples autochtones. Cependant, dans ce rappel, il effleure à peine l'histoire des pensionnats (Peelman 1992, 31-32). La CVR a libéré une parole choquante, en révélant douloureusement du même coup une crise de confiance entre les peuples autochtones et les Églises. La théologie doit en prendre acte. Les pensionnats étaient intrinsèquement abusifs: non seulement par les abus sexuels, physiques et autres perpétrés contre des personnes, mais à leur racine même, qui est celle du colonialisme et de l'assimilation à la société canadienne. Des Églises y ont participé, en partie parce qu'elles souscrivaient à ce colonialisme à leur manière. Et elles y souscrivaient en raison d'une vision du monde, d'un ordre socio-symbolique. Comment réparer, sinon en assumant aussi la tâche de la théologie telle qu'énoncée par Ched Myers: déconstruire cet ordre qui oriente discours et pratiques (non seulement religieux du passé mais aussi sociaux et contemporains) pour le remplacer par d'autres schèmes socio-symboliques libérateurs (Myers 2008; Belo 1981). Cet ordre persiste au Canada et prend des formes séculières. Ainsi, beaucoup plus de jeunes Autochtones sont placés

10. Je reprends et développe à ma manière une idée présentée par Michel Andraos dans un autre texte du présent recueil. 
hors de leurs communautés par les services de protection de la jeunesse à travers le pays, beaucoup plus qu'il n'y en a jamais eu dans les pensionnats; comme en témoignent nos gouvernements actuels, le racisme systémique rend difficile de prendre au sérieux les appels à l'aide concernant les femmes autochtones assassinées, disparues ou agressées sexuellement; et les titres ancestraux sur des territoires protégés par des traités continuent d'être bafoués à l'échelle du continent nord-américain. Nous devons critiquer cet ordre socio-symbolique, à défaut de quoi il ne fait que contaminer nos théologies. Comme l'écrit Denise Nadeau:

Le rôle des Églises dans la création de la Commission de vérité et de réconciliation a permis à ces dernières d'assumer une partie de la responsabilité du génocide culturel que les écoles ont contribué à perpétrer. Toutefois, le défi que devront relever les chrétiens immigrants et les Églises est double: il s'agit de rejeter non seulement la théologie archaïque des missionnaires du passé, mais également la culture invisible attachée à l'identité blanche, qui reste omniprésente dans l'essentiel de la théologie dominante, au Canada et au Québec. Faute de quoi, il nous sera impossible de nous reconstituer moralement, de rétablir un lien constructif avec nos propres traditions chrétiennes et de devenir des alliés de façon à nous libérer des chaînes de la suprématie blanche et de la domination impériale. (Nadeau 2012, 434)

\section{Une imagination morale et créatrice face à l'héritage des pensionnats}

Un des effets de la voie dialogale auparavant évoquée serait de surmonter la polarisation perçue par Niezen à la CVR; selon lui, les récits des différents acteurs des pensionnats se trouveraient simplifiés en deux catégories: celle des victimes et celles des bourreaux. Cette situation renforce ce que l'historien Brieg Capitaine appelle l' " incapacité à épouser la nouvelle narration des pensionnats ", qu'il perçoit dans la production théologique des oblats du Canada (Capitaine 2015, 126) ${ }^{11}$. Capitaine note que les Églises autres que catholiques, concernées par les pensionnats, ont su dépasser une certaine posture défensive et s'engager dans une créativité théologique et morale. Il redoute qu'à défaut d'emboîter le pas, l'héritage des oblats ne s'abime définitivement dans le ressentiment réciproque des oblats et de leurs accusateurs autochtones. Cela n'est pas vrai uniquement pour les

11. L'auteur met en évidence la discrétion du thème autochtone dans la revue, qui devient encore plus effacé à partir du moment où commencent les dénonciations des Autochtones à propos des pensionnats. 
oblats: une posture défensive compromet le passage à de nouveaux rapports entre l'Église catholique et les communautés autochtones. Cependant, pour rendre justice aux oblats, il faut mentionner leur support à la démarche "Retour à l'Esprit », réalisée à travers le Canada depuis plusieurs années. Elle a précisément pour but d'amener les deux camps, celui des Autochtones et celui des acteurs et actrices des pensionnats, à exprimer leurs blessures et leurs colères respectives, à engager un véritable dialogue et à s'écouter mutuellement, dans une perspective de mutuelle guérison.

Les Églises sont ainsi invitées à un travail d'imagination morale. John Paul Lederach définit celle-ci comme «la capacité d'imaginer quelque chose qui, tout en étant enraciné dans le monde réel, est capable de donner naissance à quelque chose qui n'existe pas encore ". Il affirme en outre que cette capacité «émerge avec la capacité de nous imaginer nous-mêmes en relation, la volonté d'embrasser la complexité sans se fier à la polarité dualiste, la foi en l'acte créateur, ainsi que l'acceptation du risque inhérent qui est requis pour briser le cycle de la violence et pour s'aventurer dans des voies où le changement constructif se construit. » (Lederach 2005, 29) Il y aurait lieu d'explorer les récits de vie multiples des personnes engagées dans les pensionnats pour en faire une lecture théologique sans complaisance. Il reste à faire émerger de la lumière, des postures complexes et inattendues dans ces récits, et ce, malgré l'échec, malgré l'illusion de ceux et celles qui voulaient bien faire, car il en va de passerelles et de pistes pour ce travail de longue haleine qu'est la réconciliation.

\section{Pour ne pas conclure: il faudra du temps}

Le nom de la Commission de vérité et "réconciliation » fait écho à un thème important de la tradition chrétienne. À cet égard, remontant des aspects politiques et stratégiques du projet de réconciliation à ses soubassements socio-symboliques, Corntassel en formule une autre critique: «At its core, reconciliation has a religious connotation premised on restoring one's relationship with God. In fact, most Indigenous nations don't have words for reconciliation in their languages, which is the truest test of its lack of relevance to communities. » (Corntassel 2012, 93)

Voilà une critique déstabilisante au regard de l'imaginaire biblique et chrétien. Dans l'Évangile, la réconciliation semble un impératif, au même titre que le suprême commandement de l'amour:

Mais moi, je vous dis que quiconque se met en colère contre son frère mérite d'être puni par les juges; que celui qui dira à son frère: Imbécile! mérite 
d'être puni par le sanhédrin; et que celui qui lui dira: Insensé! mérite d'être puni par le feu de la géhenne. Si donc tu présentes ton offrande à l'autel, et que là tu te souviennes que ton frère a quelque chose contre toi, laisse là ton offrande devant l'autel, et va d'abord te réconcilier avec ton frère; puis, viens présenter ton offrande... (Mt 5,22-24) $)^{12}$

Pour Paul, le Christ réconcilie l'humanité avec le Père: c'est le sens suprême de sa mission. "Au nom du Christ, nous vous en supplions, laissez-vous réconcilier avec Dieu », implore Paul (2 Co 5,20). La réconciliation, liée à l'amour, éminemment désirable, apparaît ainsi dans sa dimension théologale.

Robert J. Schreiter, un théologien étatsunien de la réconciliation, rappelle que l'opposition du Magistère catholique à la théologie de la libération était fondée sur la conception conflictuelle de la réalité sociale qu'on attachait à ce courant théologique (Schreiter 1992, 21-25). Cette critique allait culminer dans l'Instruction Libertatis Nuntius de la Congrégation pour la doctrine de la foi, en 1984 (Commission pour la doctrine de la foi 1984). Schreiter conteste cette position. Selon lui, il est tout à fait possible de reconnaître le caractère conflictuel de la réalité sans considérer le conflit comme l'indépassable réalité. Le Ressuscité est aussi indissociablement le Crucifié, dont le conflit a marqué le destin. Cette position est conforme à celle des théologies de la libération à cette époque, mais il est intéressant de la voir reprise dans une théologie de la réconciliation. Pour peu qu'on y songe, le désir d'évacuer le conflit pour y substituer un projet de réconciliation, en plus d'être absurde, relève du désir d'en finir avec la Croix et la violence systémique qu'elle manifeste.

Disciples du Réconciliateur, les chrétiens se voient confier à leur tour un ministère de réconciliation (2 Co 5,17-20). Cette mission implique qu'ils vivent personnellement dans l'esprit de la réconciliation. Mais en quoi consiste-t-il? "Vous voilà donc débarrassés du mensonge. Que chacun dise la vérité à son prochain, car nous sommes membres les uns des autres. Êtes-vous en colère? Ne péchez pas; que le soleil ne se couche pas sur votre ressentiment. (Ep 4,25-26). "Nous sommes membres les uns des autres ", certes, mais la réconciliation est liée en ceci à l'acte de vérité qui la précède.

Dans cette double perspective, la réconciliation est-elle possible avec les peuples autochtones en contexte de colonialisme et après les pensionnats? Se réconcilier est-il toujours souhaitable? Est-ce toujours opportun?

12. Traduction CEcuménique de la Bible. 
Il y a un moment pour tout et un temps pour chaque chose sous le ciel [...] un temps pour tuer et un temps pour guérir, un temps pour saper et un temps pour bâtir [...], un temps pour embrasser et un temps pour éviter d'embrasser [...], un temps pour déchirer et un temps pour coudre [...], un temps pour aimer et un temps pour haï, un temps de guerre et un temps de paix. (Qo 3,1-8)

Il est probable que le soleil ne se couchera pas de sitôt sur la colère des uns et des autres. Pour la CVR, la réconciliation est un projet de longue haleine. Il découle nécessairement d'une transformation de la société canadienne sur plusieurs plans: éducation, droit, restructuration politique, administration de la justice, aménagement territorial, transformations économiques. Entre vérité et réconciliation, il faut du temps, celui de la guérison. Nous espérons avoir esquissé quelques contributions possibles des Églises et de la théologie à ce travail qui les attend.

\section{Références}

Alfred, T. (2005), Wasáse. Indigenous Pathways of Action and Freedom, Peterborough, Ont./Orchard Park, Broadview Press.

Alfred, T. (2009), «Restitution is the Real Pathway to Justice for Indigenous Peoples", dans Aboriginal Healing Foundation (dir.), Response, Responsibility and Renewal. Canada's Truth and Reconciliation Journey (Aboriginal Healing Foundation Research Series), Ottawa, Aboriginal Healing Foundation, p. 181-187.

Andraos, M. E. (2015), "Practices of Plural Spiritualities in a Secular Society. Circles of Reconciliation with Aboriginal Peoples in Quebec ", dans R. J. Schreiter, dir., Plural Spiritualities. North American Experiences, Washington, The Council for Research in Values and Philosophy, p. 91-109.

BAum, G. (2014), «Les Églises canadiennes se repentent de leur identification avec le colonialisme ", Théologiques, 22/1, p. 101-109.

Belo, F. (1981), A Materialist Reading of the Gospel of Mark, Maryknoll, Orbis Books.

Capitaine, B. (2015), "La signification de la mission et des pensionnats indiens dans la revue Kerygma (1967-2004) », Études d'histoire religieuse, 81, p. 126-140. 
COMMISSION DE VÉRITÉ ET RÉCONCILIATION DU CANAda (2015a), Honorer la vérité, réconcilier pour l'avenir. Sommaire du rapport final de la Commission de vérité et réconciliation du Canada, Montréal/ Kingston/London/Chicago, McGill/Queen's University Press.

(2015b), Ce que nous avons retenu. Les principes de la vérité et de la réconciliation, s.l.

(2015c), Les survivants s'expriment. Un rapport de la Commission de vérité et de réconciliation du Canada (Extraits des témoignages de survivants entendus à la Commission de vérité et réconciliation du Canada).

(2015d), Pensionnats du Canada. L'histoire, partie 1 : des origines à 1939, Montréal/Kingston/London/Chicago, McGill-Queen's University Press.

(2105e), Pensionnats du Canada. L'histoire, partie 2: de 1939 à 2000, Montréal / Kingston / London / Chicago, McGill-Queen's University Press.

(2015f), Pensionnats du Canada. L'expérience inuite et nordique, Montréal / Kingston / London/Chicago, McGill-Queen's University Press.

(2015g), Pensionnats du Canada. L'expérience métisse, Montréal/ Kingston/London/Chicago, McGill-Queen's University Press.

(2015h), Pensionnats du Canada. Enfants disparus et lieux de sépulture non marqués, Montréal/Kingston/London/Chicago, McGillQueen's University Press.

(2015i), Pensionnats du Canada. Les séquelles, Montréal/ Kingston/London/Chicago, McGill-Queen's University Press.

(2015j), Pensionnats du Canada. La réconciliation, Montréal/ Kingston/London/Chicago, McGill-Queen's University Press.

(2015k), Appels à l'action, Winnipeg.

Commission pour la Justice ET LA PAIX - Conférence des ÉvêQues CATHoliques du CanAda (2016), "La "doctrine de la découverte" et la terra nullius. Réaction catholique ", disponible à <http://www.cccb.ca/ site/images/stories/pdf/reaction $\% 20$ catholique $\% 20-\% 20$ doctrine $\% 20$ de $\% 201$ la $\% 20$ decouverte $\% 20$ et $\% 20$ tn.pdf>.

CONGRÉGATION POUR LA DOCTRINE DE LA FOI (1984), Instruction sur quelques aspects de la théologie de la libération Libertatis nuntius.

CONSEIL ÉCONOMIQUE ET SOCIAL - INSTANCE PERMANENTE SUR LES QUESTIONS AUTOCHTONES (2010a), «Étude préliminaire des conséquences 
pour les peuples autochtones de la construction juridique internationale connue sous le nom de doctrine de la découverte ", New York, Organisation des Nations Unies, art. 12.

(2010b), «Statement by Permanent Observer Mission of the Holy See Economic and Social Council, 9th session of the Permanent Forum on Indigenous Issues On Agenda Item 7: Discussion on the reports "Impact on Indigenous Peoples of the International Legal Construct Known as the Doctrine of Discovery, Which Has Served as the Foundation of the Violation of their Human Rights" et "Indigenous Peoples and Boarding Schools: A Comparative Study" ", New York, Organisation des Nations Unies.

Corntassel, J. (2012), «Re-envisioning Resurgence. Indigenous Pathways to Decolonization and Sustainable Self-determination ", Decolonization: Indigeneity, Education \& Society, 1, p. 86-101.

Corntassel, J., CHAw-Win-Is et T’LAKWAdZi (2009), «Indigenous Storytelling, Truth-telling, and Community Approaches to Reconciliation ", English Studies in Canada, 35, p. 137-159.

Corntassel, J. et C. Holder (2008), «Who’s Sorry Now? Government Apologies, Truth Commissions, and Indigenous Self-determination in Australia, Canada, Guatemala, and Peru ", Human Rights Review, 9, p. 468 .

Crosby, D. (1991), «Présentation des excuses de la Conférence oblate aux Premières Nations du Canada », s.l., s.é., disponible à <http://www.cccb. $\mathrm{ca} /$ site/images/stories/pdf/excuses_oblates_francais.pdf $>$.

Deloria Jr, V. (2003), God is Red. A Native View of Religion, Golden, Fulcrum.

Donaldson, L. E. (2002), «The Breasts of Columbus. A Political Anatomy of Postcolonialism and Feminist Religious Discourse", dans L. E. Donaldson et P.-L. Kwok, dir., Postcolonialim, Feminism \& Religious Discouse, New York/London, Routldege, p. 41-61.

Harper, S. B. (2008), Présentations d'excuses aux anciens élèves des pensionnats indiens: 11 juin 2008, disponible à <http://www.aadnc-aandc. gc.ca/fra/1100100015644/1100100015649>.

IAcовucci, F. (2006), "Convention de règlement relative aux pensionnats indiens. Annexe N: Mandat de la Commission de vérité et réconciliation ».

James, M. (2010), «Uncomfortable Comparisons. The Canadian Truth and Reconciliation Commission in International Context ", Les ateliers de l'éthique/The Ethics Forum, 5, p. 23-35. 
Kidwell, C. S., H. Noley et G. E. Tinker (2001), A Native American Theology, Maryknoll, Orbis Books.

Leblanc, T. (2016), "Walking in Reconciled Relationships ", Consensus, 37, disponible à <http://scholars. wlu.ca/cgi/viewcontent.cgi? article=133 4\&context=consensus $>$, p. 4.

Leblanc, T. et J. Leblanc (2011), «NAIITS Contextual Mission, Indigenous Context », Missiology: An International Review, 39, p. 87-100.

Lederach, J. P. (2005), The Moral Imagination. The Art and Soul of Building Peace, Oxford, Oxford University Press.

Macdonald, M. (2016), "What the TRC Reveals About the Churches ", Consensus, 37, disponible à <http://scholars.wlu.ca/consensus/vol37/ iss $1 / 6>$.

Mackay, S. (2003), "An Aboriginal Perspective on the Integrity of Creation ", dans R. S. GotTlieb, dir., This Sacred Earth. Religion, Nature, Environment, New York/London, Routledge, p. 175-179.

Myers, C. (2008), Binding the Strong Man. A Political Reading of Mark's Story of Jesus, Maryknoll, Orbis Books.

Nadeau, D. (2012), «Relation et responsabilité. Vers un processus de réconciliation ", Théologiques, 20, p. 419-452.

Niezen, R. (2013), Truth and Indignation. Canada's Truth and Reconciliation Commission on Indian Residential Schools (Teaching culture: UTP ethnographies for the classroom), Toronto, University of Toronto Press.

Organisation des Nations Unies (2007), Déclaration sur les droits des peuples autochtones. New York, Organisation des Nations Unies.

Pape François, Participation à la $\mathrm{II}^{\mathrm{e}}$ rencontre mondiale des mouvements populaires, Santa Cruz de la Sierra, 9 juillet 2015.

Peelman, A. (1992), Le Christ est Amérindien. Une réflexion théologique sur l'inculturation du Christ parmi les Amérindiens du Canada, Ottawa, Novalis.

Roussel, J.-F. (2016), "Lacombe's Chart. Imperialism and Spiritual Exile in the Indian Residential Schools of Canada ", dans Gerald Boodoo, dir., Religion, Human Dignity and Liberation (World Forum on Theology and Liberation), Sao Leopoldo, Oikos, p. 209-223.

[s.d.], «Les réactions des Églises devant l'histoire des pensionnats autochtones (1969 - 2015). Entre résistances et engagement pour la réconciliation », dans M.-P. Bousquet et K. HeLe, dir., Les brûlés 
de l'âme. Les pensionnats autochtones au Québec, Toronto, Queens University Press (à paraître).

(2004), L'Esprit est amérindien. Quand la religion amérindienne rencontre le christianisme, Montréal, Médiaspaul (Spiritualités en dialogue 3).

Saint-SiÈge (2009), "Communiqué of the Holy See Press Office», dans Aboriginal Healing Foundation, dir., Response, Responsibility and Renewal, disponible à <http://www.ahf.ca/downloads/trc2.pdf>.

Schreiter, R. J. (1992), Reconciliation. Mission and Ministry in a Changing Social Order (The Boston Theological Institute series), Maryknoll/Cambridge, Orbis Books/Boston Theological Institute.

Simpson, L. (2011), Dancing on our Turtle's Back. Stories of Nishnaabeg Re-creation, Resurgence and a New Emergence, Winnipeg, Arbeiter Ring Publishing.

Treat, J. (1996), Native and Christian. Indigenous Voices on Religious Identity in the United States and Canada, New York, Routledge.

Weaver, J. (1998), Native American Religious Identity. Unforgotten Gods, Maryknoll, Orbis Books.

\section{Résumé}

La Commission de vérité et réconciliation du Canada (CVR) a traité abondamment du rôle des Églises dans le système des pensionnats autochtones et de leurs responsabilités pour surmonter cet héritage. La théologie est interpellée elle aussi par le rapport final de la CVR et par les implications de la "réconciliation ". Après avoir résumé l'histoire des pensionnats et expliqué l'origine de la CVR, nous rendons compte de ses résultats. Nous exposons les demandes que la CVR adresse spécifiquement aux Églises et aux coalitions œcuméniques. Nous examinons certaines critiques et mises en garde exprimées avant et pendant la CVR concernant le projet de réconciliation, les risques liés au mandat de la CVR et les aspects communicationnels des audiences. Nous engageons ensuite une réflexion théologique sur le thème de la réconciliation. En conclusion, nous proposons quelques pistes de réflexion pour le travail de la théologie après la CVR. 


\section{Abstract}

The Truth and Reconciliation Commission of Canada (TRC) has extensively discussed the role of churches in the residential school system and their responsibilities in overcoming this legacy. Theology is also challenged by the TRC's final report and the implications of "reconciliation". After summarizing the history of the residential schools and explaining the origin of the TRC, we report on its outcomes. We outline the requests that the TRC specifically addresses to churches and ecumenical coalitions. We review some of the criticisms and caveats expressed before and during the $T R C$ regarding the reconciliation project, the risks associated with the TRC's mandate, and the communication aspects of the hearings. We then engage in theological reflection on the theme of reconciliation. In conclusion, we propose some ideas for the work of theology after the CVR. 